\title{
Atypical Wernicke's Encephalopathy with Remarkable Cerebellar Lesions on Diffusion-Weighted MRI
}

\author{
Hyun-Ah Kim ${ }^{a}$ Hyung Lee ${ }^{a, b}$ \\ Department of Neurology, ${ }^{a}$ Keimyung University School of Medicine, and ${ }^{b}$ Brain Research Institute, \\ Keimyung University School of Medicine, Daegu, South Korea
}

Dear Sir,

Typical MRI findings of Wernicke's encephalopathy (WE) are symmetrical hyperintense lesions in the medial thalamus, mamillary body, periaqueductal gray matter, and floor of the fourth ventricle $[1,2]$. We report a patient with WE who showed an unusual location of high signal lesions with extensive cerebellar involvements on diffusion-weighted MRI. WE should be considered in the differential diagnosis of high signal lesions in the cerebellum on diffusion-weighted MRI.

\section{Case Report}

A 56-year-old man was admitted to the hospital for altered consciousness. His history included drinking 2-3 liters per day of grain wine at a $6 \%$ alcohol content for 8 years. No other vascular risk factor was found. Both hands trembled and he had walked with an unsteady gait for the preceding year. He was lethargic for 8 days prior to admission, but did respond to simple commands. At the time of admission he was deeply stuporous and he could not withdraw from painful stimuli. Neurological examination revealed a nearly absent horizontal oculocephalic response. Serum electrolytes and osmolarity were normal. Diffusion-weighted images (DWI) of the initial brain MRI performed on the first hospital day showed diffuse hyperintense lesions in the bilateral cerebellar hemispheres extending into the posterior hemi- sphere and posterior vermis, simulating acute bilateral cerebellar infarctions (fig. 1A). An initial diagnosis was made based on a history of chronic alcohol abuse, with typical symptoms that included altered mental state and ophthalmoplegia, and combined lesions on bilateral thalamus and periaqueductal gray matter on DWI (fig. 1B). Immediate administration of $300 \mathrm{mg}$ thiamine commenced. On the second hospital day, he became drowsy and did respond to simple commands. Mild horizontal gaze palsy, gaze-evoked nystagmus, and limb dysmetria were evident. A follow-up DWI performed 8 days after onset showed that the previously high signal intensities in the cerebellum (fig. 1A), medial thalamus, and periaqueductal gray matter (fig. 1B) had mostly resolved. Follow-up $\mathrm{T}_{2}$-weighted images showed no high signal intensity, but severe atrophy of the cerebellar vermis and anterior hemisphere were evident (fig. 1A). MR angiography showed no definite stenosis or occlusion in the vertebrobasilar circulation. Over several days of hospitalization, he regained alertness, but showed considerable memory impairment and gait ataxia.

\section{Discussion}

It is well known from autopsy that the anterior-superior vermis or anterior hemisphere are affected in more than half of patients with WE. A recent study [3] showed that 13 of 15 consecutive patients with WE showed cerebellar vermian atrophy on initial or follow-up MRI, but none of the patients showed high signal intensity in the cerebellum on MRI. There have only been three reports [4-6] of high signal abnormalities in the cerebellum on MRI during the acute stage of WE, such as were exhibited by our patient. Furthermore, in previous reports lesions were confined to the superior portion of the cerebellar vermis or anterior hemisphere. To our knowledge, this is the first report of WE with extensive bilateral cerebellar involvement extending into the posterior hemisphere and posterior vermis.

Because acute ischemic stroke is the most common cause of high signal lesion on DWI, as pointed out by Lovblad et al. [7], an embolic cause of cerebellar infarction should be considered in our case. Furthermore, high signals on DWI due to embolic infarction sometimes dissolve spontaneously due to reperfusion or pseudonormalization. But the resolution of an infarct due to rapid recanalization is limited by its small volume in human studies $[8,9]$. Moreover, improvement in symptoms in our patient was not so rapid or predominant as to imply reperfusion of a large cerebellar infarct. Absence of cerebellar hyperintensity on follow-up $\mathrm{T}_{2}$-weighted image speaks against the possibility of pseudonormalization.

\section{KARGER}

Fax +41613061234 E-Mail karger@karger.ch www.karger.com www.karger.com/ene
Hyung Lee, MD

Department of Neurology, Keimyung University School of Medicine 194 Dongsan dong

700-712 Daegu (South Korea)

Tel. +82 53250 7835, Fax +8253250 7840, E-Mail hlee@dsmc.or.kr 

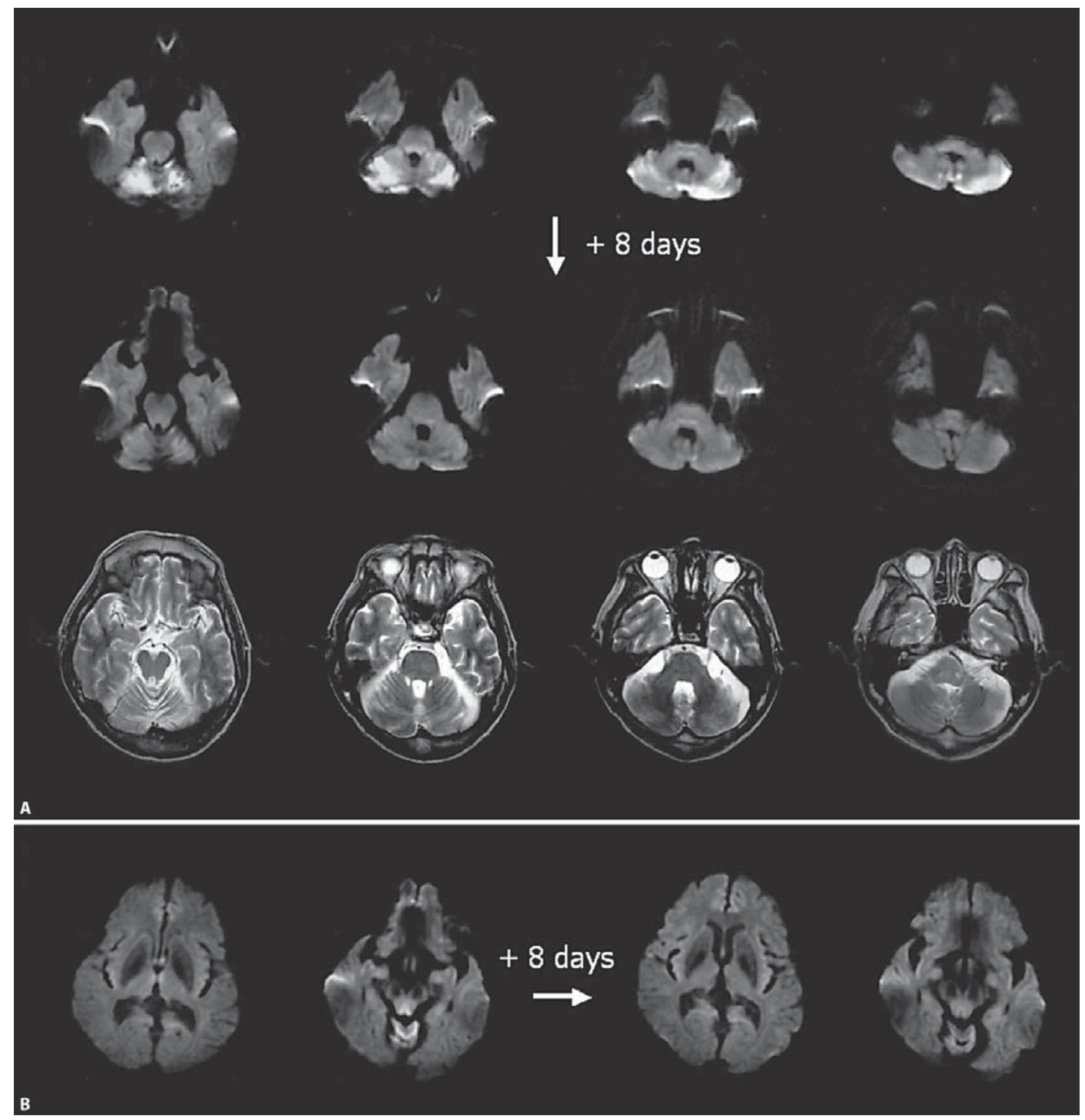

Fig. 1. A DWI of the initial brain MRI showing high signal intensities bilaterally in the cerebellum and extending into the posterior hemisphere and posterior vermis. The lesions had mostly resolved on DWI in a follow-up MRI after thiamine administration. $\mathrm{T}_{2}$-weighted images showed no high signal intensity, but there was severe atrophy of the anterior cerebellar hemisphere and vermis on follow-up MRI. B DWI of the initial MRI showed hyperintense lesions in the medial thalamus and periaqueductal gray matter, consistent findings with WE. These had resolved mostly on follow-up MRI after thiamine administration. 
It is also possible that extensive cerebellar involvement in our patient is associated with progressive alcoholic cerebellar degeneration not related to $\mathrm{WE}$, since $\mathrm{WE}$ and cerebellar degeneration commonly occur in the same individual along with chronic alcoholism, while both conditions can cause gait ataxia. However, considering the classic symptoms of mental alteration and ophthalmoplegia, typical MRI findings with high signal bilateral thalamic and periaqueductal gray matter lesions, and rapid clinical and radiological improvement after thiamine administration, WE-related cerebellar involvement with high signal lesions on DWI is considered a more likely pathogenesis. Moreover, to the best of our knowledge, no prior reports of alcoholic cerebellar degeneration have described high signal cerebellar lesions.

High signal intensity on DWI in acute WE may reflect necrosis, apoptosis, and cellular edema associated with a reduction in the activity of intermediate enzymes of carbohydrate metabolism [10]. It is difficult to know exactly why cerebellar lesions are more prominent than lesions in the medial thalamus and periaqueductal gray matter. Presumably, chronic neural injury due to WE-related metabolic change may have caused degeneration in the commonly affected areas more than the cerebellum, so that cerebellar lesions would then appear relatively prominent.

In conclusion, WE can be associated with various findings on MR images, thus clinical features are essential for diagnosis. Clinicians should remain aware of the possibility of WE in patients with typical clinical manifestations, even when atypical MR findings are present.

\section{References}

1 Opdenakker G, Gelin G, De Surgeloose D, Palmers Y: Wernicke encephalopathy: MR findings in two patients. Eur Radiol 1999;9: 1620-1624.

2 Gallucci M, Bozzao A, Splendiani A, Masciocchi C, Passariello R: Wernicke encephalopathy: MR findings in five patients. AJNR Am J Neuroradiol 1990;11:887-892.

3 Park SH, Kim M, Na DL, Jeon BS: Magnetic resonance reflects the pathological evolution of Wernicke encephalopathy. J Neuroimaging 2001;11:406-411.
4 Bae SJ, Lee HK, Lee JH, Choi CG, Suh DC: Wernicke's encephalopathy: atypical manifestation at MR imaging. Am J Neuroradiol 2001;22:1480-1482.

5 Murata T, Fujito T, Kimura H, Omari M, Itoh H, Wada Y: Serial MRI and (1) H-MRS of Wernicke's encephalopathy: report of a case with remarkable cerebellar lesions on MRI. Psychiatry Res 2001;108:49-55.

6 Rugilo CA, Uribe Roca MC, Zurru MC, Capizzano AA, Pontello GA, Gatto EM: Proton MR spectroscopy in Wernicke encephalopathy. Am J Neuroradiol 2003;24:952-955.

7 Lovblad KO, Laubach HJ, Baird AE, Curtin F, Schlaug G, Edelman RR, Warach S: Clinical experience with diffusion-weighted $M R$ in patients with acute stroke. AJNR Am J Neuroradiol 1998;19:1061-1066.

8 Fiehler J, Foth M, Kucinski T, Knab R, von Bezold M, Weiller C, Zeumer H, Rother J: Severe ADC decreases do not predict irreversible tissue damage in humans. Stroke 2002;33:79-86.

9 Kidwell CS, Alger JR, Di Salle F, Starkman S, Villablanca P, Bentson J, Saver JL: Diffusion MRI in patients with transient ischemic attacks. Stroke 1999;30:1174-1180.

10 Hazell AS, Todd KG, Butterworth RF: Mechanisms of neuronal cell death in Wernicke's encephalopathy. Metab Brain Dis 1998;13: $97-122$. 